\title{
COMPORTAMENTO DINÂMICO-MECÂNICO E RELAXAÇÕES EM POLÍMEROS E BLENDAS POLIMÉRICAS
}

\author{
Silvana Navarro Cassu
}

Divisão de Química, Instituto de Aeronáutica e Espaço, Centro Técnico Aeroespacial, Praça Mal. Eduardo Gomes, 50, 12228-904 São José dos Campos - SP

Maria Isabel Felisberti*

Instituto de Química, Universidade Estadual de Campinas, CP 6154, 13084-971 Campinas - SP

Recebido em 3/11/03; aceito em 26/8/04; publicado na web em 2/2/05

DYNAMIC MECHANICAL BEHAVIOR AND RELAXATIONS IN POLYMERS AND POLYMERIC BLENDS. Dynamic mechanical analysis (DMA) is widely used in materials characterization. In this work, we briefly introduce the main concepts related to this technique such as, linear and non-linear viscoelasticity, relaxation time, response of material when it is submitted to a sinusoidal or other periodic stress. Moreover, the main applications of this technique in polymers and polymer blends are also presented. The discussion includes: phase behavior, crystallization; spectrum of relaxation as a function of frequency or temperature; correlation between the material damping and its acoustic and mechanical properties.

Keywords: DMA; polymer; polymer blends.

\section{INTRODUÇÃO}

A análise dinâmico-mecânica (DMA) ou análise termodinâmicomecânica (DMTA) tem como um dos principais objetivos relacionar as propriedades macroscópicas, tais como as propriedades mecânicas, às relaxações moleculares associadas a mudanças conformacionais e a deformações microscópicas geradas a partir de rearranjos moleculares ${ }^{1}$.

As propriedades mecânicas dos materiais são avaliadas a partir de uma solicitação, na forma de uma deformação ou na aplicação de uma tensão, com o monitoramento da resposta do material, expressa como tensão ou como deformação, respectivamente. Ensaios mecânicos são classificados como estáticos, uma vez que se aplica ao material uma tensão ou deformação constante, ou a taxas constantes. Estes experimentos são destrutivos, já que uma de suas finalidades é a determinação de propriedades limite do material.

A análise dinâmico-mecânica consiste, de modo geral, em se aplicar uma tensão ou deformação mecânica oscilatória, normalmente senoidal, de baixa amplitude a um sólido ou líquido viscoso, medindo-se a deformação sofrida por este ou a tensão resultante, respectivamente, sob variação de frequiência ou de temperatura ${ }^{2}$.

O comportamento mecânico ou dinâmico-mecânico de um material será governado por sua viscoelasticidade, que será função do tipo de ensaio e de solicitação aplicados. Dependendo da resposta ao estímulo mecânico, o material pode ser classificado como elástico ou viscoso. Para um sólido perfeitamente elástico ou Hookiano a deformação é proporcional à tensão aplicada e vice-versa, segundo a lei de Hooke. Um material viscoso ideal obedece à lei de Newton, que estabelece que a tensão e a taxa de cisalhamento estão relacionadas através de uma característica intrínseca que é a viscosidade ${ }^{1,2}$. Materiais poliméricos apresentam comportamento mecânico intermediário ao elástico e ao viscoso, sendo denominados viscoelásticos. A contribuição elástica e viscosa para o comportamento mecânico do polímero depende da temperatura e da escala de tempo do experimento.

O comportamento viscoelástico de materiais poliméricos pode ser compreendido mais facilmente a partir de ensaios mecânicos.

*e-mail: misabel@iqm.unicamp.br
Resultados típicos de ensaios de fluência são mostrados na Figura 1, e se relacionam a testes realizados sob cisalhamento, sendo que efeitos similares são observados em ensaios no modo de tração. Quando uma tensão constante de cisalhamento, $\sigma_{1}$ ou $\sigma_{2}$, é aplicada a uma amostra viscoelástica observa-se que a deformação, $\gamma_{1}(\mathrm{t})$ ou $\gamma_{2}(\mathrm{t})$, é dependente do tempo (Figura 1a). Se a deformação a um determinado tempo, por exemplo $t_{\mathrm{a}}$, após a aplicação da tensão é mostrada em um gráfico como função da tensão, observa-se que deformação $\gamma\left(t_{\mathrm{a}}\right)$ aumenta linear e proporcionalmente à tensão aplicada, Figura $1 \mathrm{~b}$. Para um tempo maior, $t_{b}$ após a aplicação da tensão, a deformação $\gamma\left(\mathrm{t}_{\mathrm{b}}\right)$ também aumentará linearmente com a tensão. Assim, se em um tempo arbitrário $\mathrm{t}$ a deformação relativa às tensões aplicadas forem $\gamma_{1}(\mathrm{t})$ e $\gamma_{2}(\mathrm{t})$, então ${ }^{3}$

$$
\frac{\gamma_{1}(t)}{\sigma_{1}}=\frac{\gamma_{2}(t)}{\sigma_{2}}
$$

As deformações nos dois experimentos no mesmo tempo são proporcionais à tensão imposta. Este fato conduz à definição da compliança, $\mathrm{J}(\mathrm{t})$, no tempo t:

$$
J(t)=\frac{\gamma_{1}(t)}{\sigma_{1}}=\frac{\gamma_{2}(t)}{\sigma_{2}}
$$

A Equação (2) pode ser escrita de uma forma geral como:

$J(t)=\frac{\gamma(t)}{\sigma}$

Materiais poliméricos exibem viscoelasticidade linear em ensaios de fluência quando se aplicam tensões que produzem deformações abaixo de $0,005^{3}$.

Quando a compliança é medida em várias décadas de tempo e mostrada em um gráfico em função do $\log t$ tem-se, em geral, o formato da curva mostrado na Figura 1c. Para ensaios realizados em tempos muito curtos ou muito longos $\mathrm{J}(\mathrm{t})$ mostrará pouca ou nenhuma dependência do tempo, mostrando um formato sigmoidal, que será completamente visível quando o experimento é realizado em 
(a)

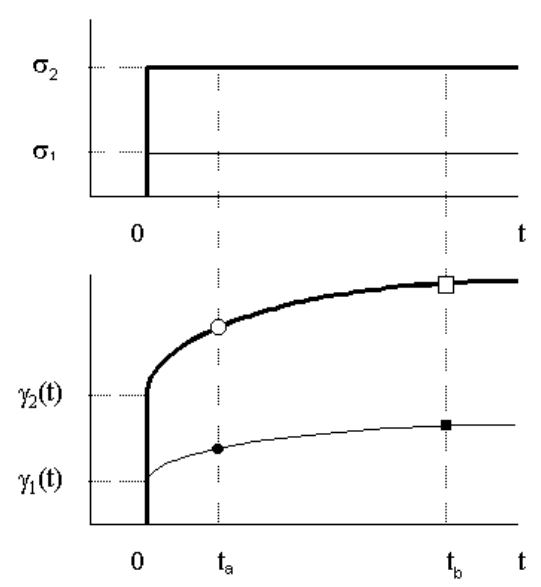

(b)

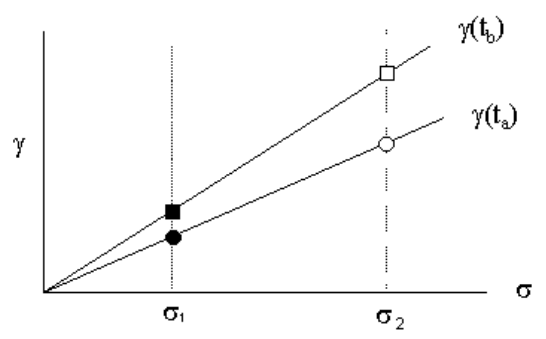

(c)

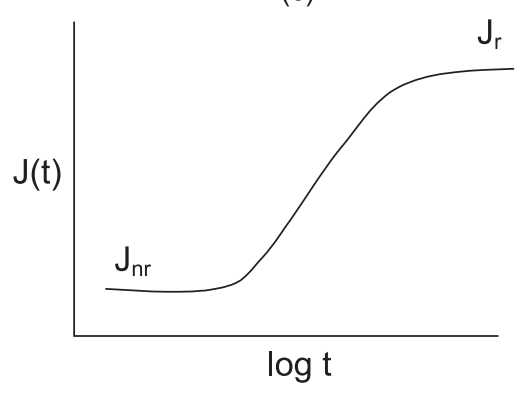

(d)

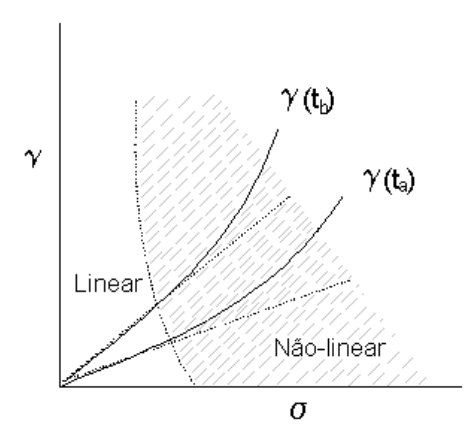

Figura 1. Viscoelasticidade linear em ensaio de fluência ("creep"): (a) tensão constante, $\sigma_{1}$ ou $\sigma_{2}$, aplicada em $t=0$ levando à deformação dependente do tempo, $\gamma_{I}(t)$ e $\gamma_{2}(t)$, respectivamente; (b) deformação linear, $\gamma(t a)$ e $\gamma(t b)$, nos tempos ta e tb, causada pela tensão aplicada, $\sigma_{1}$ ou $\sigma_{2}$, respectivamente; (c) dependência de $J(t)$ com o log do tempo durante uma relaxação completa, segundo a Equação (3). $J_{n r}$ e $J_{r}$ são as complianças para o sistema não relaxado e relaxado, respectivamente; (d) regiões de deformação linear e não-linear nos tempos $t_{a^{\prime}} \gamma\left(t_{a}\right)$, e $t_{b}, \gamma\left(t_{b}\right)$ várias décadas de tempo ${ }^{3}$. As regiões de viscoelasticidade linear e não-linear na curva de deformação em função da tensão são mostradas na Figura 1d. Deve-se notar que na região linear a compliança é independente da tensão aplicada, o que não ocorre na região de viscoelasticidade não-linear, onde cada valor de tensão conduzirá a um valor de deformação dependente do tempo. Quando o ensaio é realizado na região de viscoelasticidade não-linear, a amostra fluirá mais rápido (linha contínua) que o predito para região linear de viscoelasticidade (linha tracejada) ${ }^{3}$.

Um experimento alternativo ao ensaio de fluência é o ensaio de relaxação de tensão, no qual uma deformação constante de cisalhamento, $\gamma_{1}$, é aplicada em t=0 e a tensão, $\sigma(\mathrm{t})$, necessária para manter a deformação aplicada constante é observada. A tensão diminui com o tempo de experimento. Em dois experimentos conduzidos a diferentes deformações, $\gamma_{1}$ e $\gamma_{2}$, observa-se a um dado tempo $t$ a relação:

$$
\frac{\sigma_{1}(t)}{\gamma_{1}}=\frac{\sigma_{2}(t)}{\gamma_{2}}
$$

As tensões em dois experimentos no mesmo tempo t serão proporcionais às deformações impostas. Este fato conduz à definição do módulo de relaxação de tensão no tempo t:

$G(t)=\frac{\sigma(t)}{\gamma}$

O módulo G é equivalente ao módulo de Young E, quando a deformação de cisalhamento é substituída por deformação no modo de tração ou compressão. A definição do módulo G conforme a Equação (5) é válida para regiões de vicoelasticidade linear que compreendem deformações abaixo de $0,005^{3}$.

Comparando-se as Equações (3) e (5) nota-se que o módulo é o inverso da compliança. O mesmo comportamento observado para $\mathrm{J}(\mathrm{t})$ quando o experimento é conduzido em um grande número de décadas de tempo será observado para G(t), ou seja, observa-se uma curva sigmoidal.

\section{Ensaio dinâmico-mecânico}

A análise dinâmico-mecânica permite a separação da contribuição elástica e viscosa em materiais viscoelásticos, em função tanto da temperatura como do tempo.

A análise dinâmico-mecânica pode ser conduzida no modo de oscilação forçada (Figura 2a) ou no modo de oscilação livre (Figura 2b). No modo de oscilação forçada uma determinada amplitude é imposta ao material, variando-se a temperatura ou a frequêencia do experimento e mantendo-se a amplitude constante durante todo o ensaio. Na Figura 2c encontra-se uma representação esquemática do que ocorre com uma amostra quando submetida a uma tensão oscilatória no modo de tração. A medida que a roldana gira, a amostra presa a ela é estirada e comprimida uniaxialmente e de forma oscilatória. No experimento no modo de oscilação livre, a amostra é submetida a uma deformação em sua frequiência natural de ressonância, e registra-se o decaimento da amplitude em função do tempo $^{3-5}$ (Figura 2b).

\section{Princípios da técnica dinâmico-mecânica}

Supondo que uma amostra é submetida a uma tensão senoidal $(\sigma)$

$\sigma(t)=\sigma_{0} \cdot \operatorname{sen}(\omega t)$

sua resposta será uma deformação também senoidal $(\varepsilon)$ 
$\varepsilon(t)=\varepsilon_{0} \cdot \operatorname{sen}(\omega t+\delta)$

onde $\omega$ é a frequiência angular da tensão oscilatória à qual a amostra é submetida, $\mathrm{t}$ - tempo, $\delta$ - ângulo de defasagem ou atraso entre a tensão e a deformação máxima, e $\sigma_{0}$ e $\varepsilon_{0}$ são as amplitudes da tensão e da deformação, respectivamente, como mostrado na Figura 2a. Rescrevendo a Equação (7) obtém-se:

$\varepsilon(t)=\varepsilon_{0} \cdot \operatorname{sen}(\omega t) \cdot \cos \delta+\varepsilon_{0} \cdot \cos (\omega t) \cdot \operatorname{sen} \delta$

No caso de materiais perfeitamente elásticos a tensão aplicada e a deformação gerada na amostra estão em fase, e o ângulo de defasagem, $\delta$, será zero. Neste caso, a Equação (8) se reduz a:

$\varepsilon(\mathrm{t})=\varepsilon_{0} \cdot \operatorname{sen}(\omega \mathrm{t})$

e, portanto, a tensão aplicada e a deformação variam em função do $\operatorname{sen}(\omega t)^{2,6-7}$.

Materiais completamente viscosos apresentarão ângulo máximo de defasagem $\left(90^{\circ}\right)$ entre a tensão aplicada e a deformação gerada, e a Equação (8) se reduzirá a:

$\varepsilon(\mathrm{t})=\varepsilon_{0} \cdot \cos (\omega \mathrm{t})$

que representa a deformação completamente fora de fase em relação à tensão aplicada, variando em função de $\cos (\omega t)$ quando a amostra é submetida a uma tensão senoidal ${ }^{2,6-7}$, que é o caso de uma amostra contendo apenas a componente viscosa. Um material que responde desta forma ao estímulo é classificado como viscoso.

No caso de materiais viscoelásticos o valor de $\delta$ estará entre 0 e $90^{\circ}$, descrevendo o comportamento intermediário ao elástico e ao viscoso e a relação entre a tensão ou deformação aplicada e a resposta do material é aquela representada na Figura 2a.

Tomando-se as Equações (6) e (7), define-se o módulo de armazenamento, E' ou G' como sendo a razão entre a amplitude da componente da tensão em fase com a deformação pela amplitude da deformação:

$\mathrm{E}^{\prime}=\left(\sigma_{0} / \varepsilon_{0}\right) \cos \delta=\mathrm{E}^{*} \cdot \cos \delta$

$\mathrm{G}^{\prime}=\left(\sigma_{0} / \varepsilon_{0}\right) \cos \delta=\mathrm{G}^{*} \cdot \cos \delta$

sendo E' o módulo de elasticidade obtido em experimentos nos quais a amostra é submetida a estímulos unidirecionais (tração e compressão) e G' o módulo de cisalhamento elástico.

O módulo de armazenamento é uma medida da energia mecânica que o material é capaz de armazenar, em determinadas condições experimentais, na forma de energia potencial ou elástica ${ }^{2}$.

A razão entre a amplitude da componente da tensão fora de fase em relação à deformação pela amplitude da deformação é definida como módulo de perda, E" ou G".

$\mathrm{E}^{\prime \prime}=\left(\sigma_{0} / \varepsilon_{0}\right) \operatorname{sen} \delta=\mathrm{E}^{*} \operatorname{sen} \delta$

$\mathrm{G}^{\prime \prime}=\left(\sigma_{0} / \varepsilon_{0}\right) \operatorname{sen} \delta=\mathrm{G}^{*} \operatorname{sen} \delta$

O módulo de perda é diretamente proporcional ao calor dissipado $(\mathrm{H})$ por ciclo, de acordo com a equação:

$\mathrm{H}=\pi \mathrm{G}^{\prime} \varepsilon_{\mathrm{m}}^{2}$

onde $\varepsilon_{\mathrm{m}}$ é o valor máximo da deformação durante o ciclo ${ }^{5}$.

Essa dissipação de calor é atribuída, por exemplo, ao movimento de longos segmentos da cadeia principal, como ocorre na transição vítrea ${ }^{1-2,6-7,10}$ ou a relaxações de segmentos laterais resultantes, a)

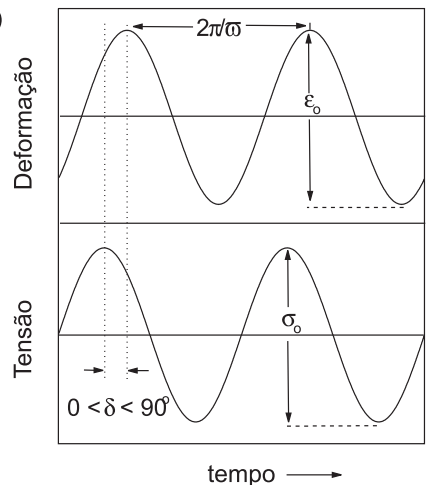

b)
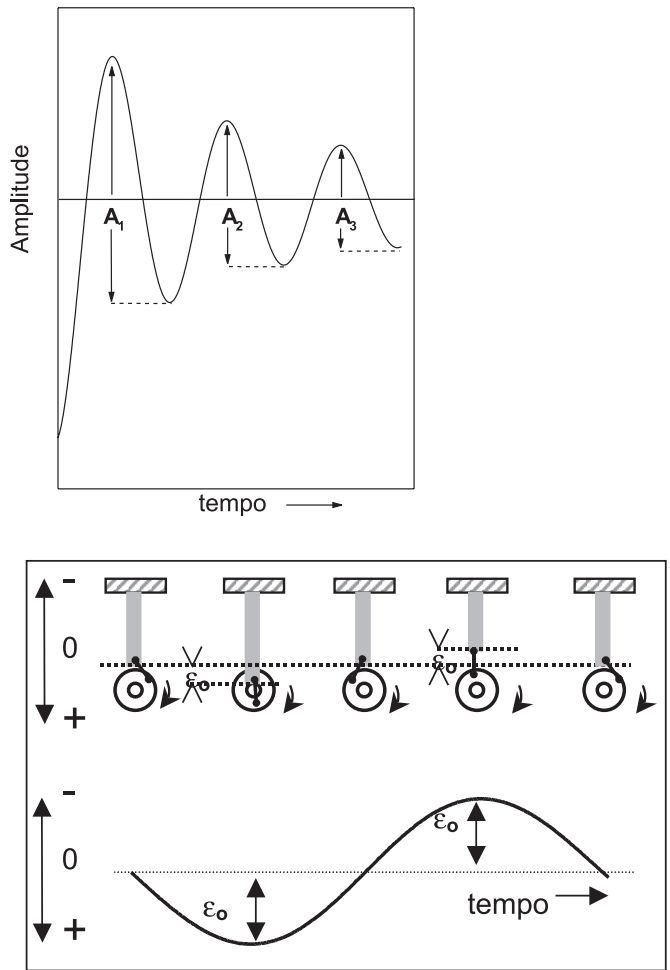

Figura 2. Ensaio dinâmico-mecânico em material viscoelástico em diferentes modos: (a) oscilação forçada e (b) oscilação livre; (c) representação da deformação sofrida por amostra viscoelástica quando submetida a uma tensão senoidal em um ensaio do tipo tração

por exemplo, de rotações em torno de ligações químicas (Figura 3).

Os módulos dinâmicos de Young, E*, e de cisalhamento, $\mathrm{G}^{*}$, podem ser expressos em notação de números complexos em suas componentes:

$\mathrm{E}^{*}=\mathrm{E}^{\prime}+\mathrm{i} \mathrm{E}^{\prime}$

$\mathrm{G}^{*}=\mathrm{G}^{\prime}+\mathrm{iG} \mathrm{G}^{\prime}$

Dividindo-se a Equação (13) pela Equação (11) tem-se:

$\frac{E^{\prime \prime}}{E^{\prime}}=\frac{E^{*} \operatorname{sen} \delta}{E^{*} \cos \delta}=\tan \delta$

onde $\tan \delta$ é denominada de fator de perda ou "damping". Essa mesma relação é valida para os parâmetros obtidos em ensaios de cisalhamento, G*, G' e G'.

$\mathrm{O}$ "damping" expressa a capacidade de um material em converter energia mecânica. Sólidos que possuem apenas a componente 
(B)

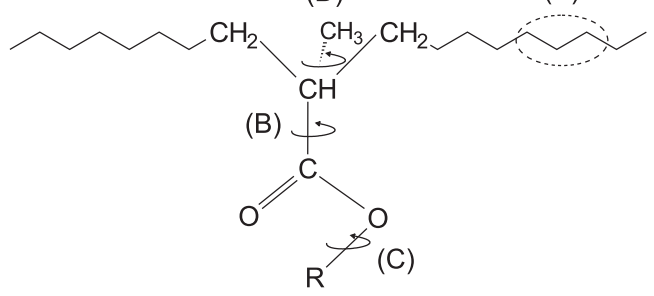

Figura 3. Representação esquemática dos possíveis modos de movimentos moleculares em relaxação secundária em polímeros vítreos: (A) pequenos movimentos da cadeia principal, (B) movimentos rotacionais de grupos laterais, (C) movimentos localizados internos de uma das unidades que faz parte de um grupo lateral ligado à cadeia principal

elástica apresentam $\tan \delta$ igual a zero. Exemplos de materiais que apresentam praticamente apenas a componente elástica são os metais e o quartzo. Polímeros, por outro lado, apresentam valores de $\delta$ da ordem de alguns graus: em certas faixas de temperatura, por exemplo, na região de transição vítrea, $\delta$ aproxima-se de $30^{\circ}{ }^{3}$.

\section{Dependência do comportamento dinâmico-mecânico com a frequiência e a temperatura}

Em experimentos dinâmico-mecânicos conduzidos isotermicamente, quando a frequiência é variada, as cadeias poliméricas irão absorver energia em faixas de frequiência características. A absorção de energia só ocorrerá quando a escala de tempo do experimento, ditada pela frequiência, for igual ao tempo necessário para que o material sofra uma determinada relaxação, denominado tempo de relaxação. Como a absorção de energia mecânica de polímeros ocorre em uma faixa de freqüência $v(1 / v=$ tempo), isto significa que $o$ material apresenta uma distribuição de tempos de relaxação, podendo estar associado, por exemplo, a um segmento de cadeia em diferentes microambientes. Os tempos de relaxação são dependentes da temperatura, decaindo com o aumento desta. Assim, em uma situação hipotética, se uma análise é conduzida a $10 \mathrm{~Hz}$, e o tempo de relaxação de um determinado grupo a esta temperatura é de $1 \mathrm{~s}$, esta relaxação não será observada neste experimento, pois o tempo entre cada ciclo é de $0,10 \mathrm{~s}$, sendo menor que o tempo necessário para que a relaxação ocorra. Entretanto, elevando-se a temperatura a $\mathrm{T}_{2}$, na qual os tempos de relaxação são da ordem de $0,10 \mathrm{~s}$, a relaxação ocorrerá.

As relaxações normalmente estão associadas a mudanças conformacionais de grupos ou segmentos da cadeia polimérica, resultantes de rotações em torno das ligações químicas. O tempo de relaxação é uma medida da mobilidade das cadeias e depende da estrutura molecular e da temperatura. A temperatura influencia a mobilidade da cadeia polimérica e, conseqüentemente, o tempo relacionado à sua relaxação. A dependência dos módulos de armazenamento e de perda com a frequiência e com a temperatura é esquematizada na Figura 4.

O efeito da variação da freqüência e da temperatura sobre o comportamento dinâmico-mecânico de materiais poliméricos pode ser resumido da seguinte forma: 1) a baixas temperaturas ou altas freqüências o polímero comporta-se como um material vítreo e, portanto, rígido, devido aos altos tempos de relaxação comparativamente à escala de tempo do experimento. Sob estas condições o módulo de armazenamento é alto e o de perda é baixo; 2) a altas temperaturas ou baixas frequiências, os movimentos internos respondem à tensão aplicada. Dessa forma, tanto o módulo de armazenamento como o de perda são baixos, correspondendo ao comportamento viscoso; 3 ) quando a freqüência do experimento é comparável à freqüência dos

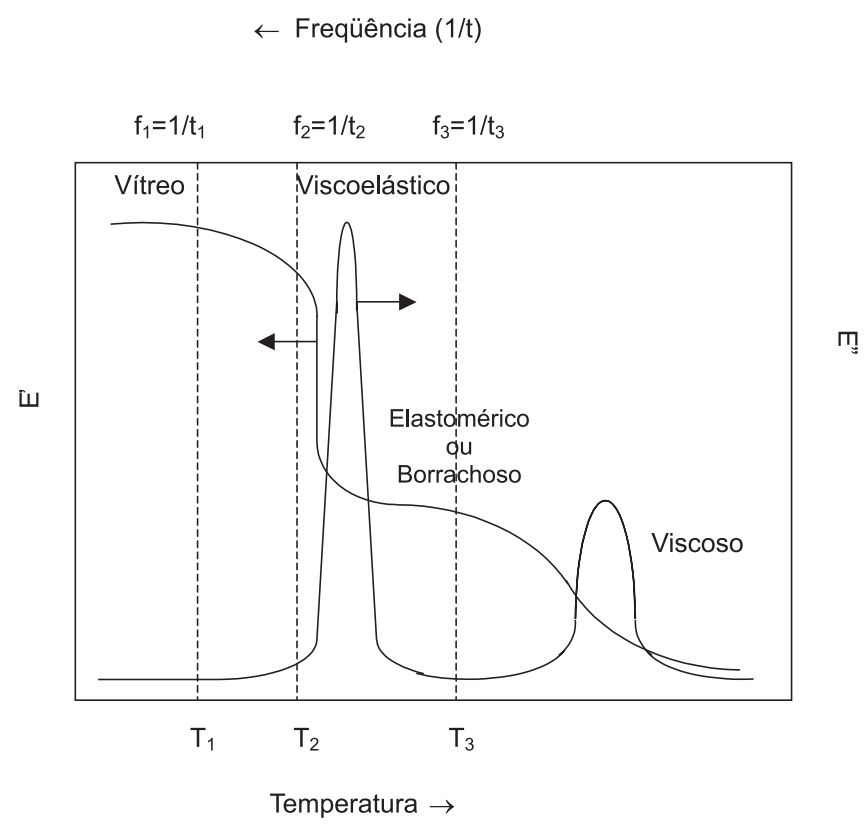

Figura 4. Dependência dos módulos de armazenamento, E', e de perda, E”, em materiais poliméricos com a variação da freqüência e da temperatura: $f_{1}>f_{2}>f_{3} e T_{1}<T_{2}<T_{3}$

movimentos internos do material na temperatura na qual é feita a medida, o módulo de armazenamento diminui com o aumento da temperatura ou com a diminuição da frequiência, e o módulo de perda exibe um máximo, correspondendo ao comportamento viscoelástico ${ }^{1}$.

As propriedades dinâmico-mecânicas de materiais poliméricos são estudadas, em geral, em uma ampla faixa de temperatura (-150 a $500{ }^{\circ} \mathrm{C}$ ) a freqüência fixa, o que permite determinar as temperaturas nas quais ocorrem as relaxações. $\mathrm{O}$ experimento contrário também pode ser executado, ou seja, a temperatura fixa e frequiência variada. Deste experimento pode ser obtido o espectro de relaxação do material, a partir do qual se obtêm todas as funções viscoelásticas, sem a necessidade de experimentos adicionais ${ }^{8,9}$. Os equipamentos comerciais operam em uma faixa estreita de freqüência, de forma que a obtenção do espectro de relaxação em uma ampla faixa de frequiência pode ser obtido a partir da aplicação do princípio de superposição tempo-temperatura. Neste caso, faz-se necessária a realização de isotermas, nas quais se varia a freqüência em uma faixa de temperatura tão ampla quanto possível ${ }^{8,9}$.

\section{Aplicações da técnica no estudo de polímeros}

A técnica dinâmico-mecânica tem sido amplamente aplicada no estudo de homopolímeros e de sistemas poliméricos mais complexos, tais como copolímeros e blendas, podendo-se obter informações diversas. A seguir, são apresentados exemplos da aplicação da técnica em sistemas poliméricos.

\section{Relaxações poliméricas}

Por meio da análise dinâmico-mecânica é possível obter informações sobre transições termodinâmicas de primeira ordem, tais como a fusão e a cristalização em condições de aquecimento, bem como transições termodinâmicas de pseudo segunda ordem, como é o caso da transição vítrea e das relaxações secundárias associadas às fases cristalina e amorfa ${ }^{6}$. A relaxação que ocorre na região de transição vítrea, também chamada de relaxação primária ou relaxação $\alpha$ para 
polímeros amorfos, é resultante dos movimentos moleculares a longas distâncias, que envolvem os segmentos da cadeia polimérica principal $^{10}$. Na região de transição vítrea, o comportamento mecânico de polímeros amorfos varia de um comportamento típico de material rígido para o de um material elastomérico. A faixa de temperatura na qual este processo ocorre depende de vários fatores, tais como a composição, a flexibilidade das cadeias, a massa molar do polímero, a presença de plastificantes, o grau de reticulação e de cristalinidade ${ }^{10-12}$. Para polímeros amorfos, que não contém carga, o módulo de armazenamento na região de comportamento vítreo é da ordem de $10^{9} \mathrm{~Pa}$ e sofre uma variação brusca da ordem de $10^{3}$ a $10^{4} \mathrm{~Pa}$ na região da transição vítrea ${ }^{2,11}$. Na Figura 5 é mostrado o comportamento dinâmico-mecânico típico de polímeros amorfos e semicristalinos em função da temperatura, exemplificado pelo poli(tereftalato de etileno) (PET) amorfo e semicristalino. A transição vítrea é caracterizada pela queda abrupta do módulo de armazenamento E' (Figura 5a) e pelos máximos das curvas de E" (Figura 5b) e $\tan \delta$ (Figura 5c) em função da temperatura. O máximo observado no módulo de perda, E", na região da transição vítrea deve-se à alta conversão de energia mecânica em calor através dos movimentos micro-Brownianos dos segmentos da cadeia principal. Como o tan $\delta$ é a razão entre os módulos de perda e armazenamento, atingirá valores máximos em regiões próximas aos máximos observados para o E", porém em maiores temperaturas. O máximo do pico da curva de E" x T corresponde à situação de máxima dissipação de energia mecânica, que na região de transição vítrea está associada à mudança do estado vítreo para o elástico.

Comparando-se as curvas do módulo de armazenamento, Figura 5a, nota-se que a curva correspondente ao PET amorfo apresenta uma queda brusca no valor do módulo na região de transição vítrea, enquanto para o PET semicristalino o módulo de armazenamento diminui em apenas uma ordem de grandeza na região da transição vítrea (entre 110 e $140^{\circ} \mathrm{C}$ ), seguido de uma queda mais brusca próxima a $200^{\circ} \mathrm{C}$ devido ao início da fusão. Nas curvas de E" x T (Figura 5b) é clara a influência da fase cristalina na largura do pico correspondente à transição vítrea, o que significa que o material semicristalino, por apresentar diferentes microambientes ${ }^{13}$ - fase amorfa, interface cristalina-amorfa e fase cristalina - apresenta uma distribuição larga de tempos de relaxação. As temperaturas correspondentes aos máximos da curva E" x T para o PET amorfo e cristalino são 93 e $118^{\circ} \mathrm{C}$, respectivamente.

Além das relaxações associadas à transição vítrea, há as relaxações secundárias referentes à fase amorfa, que ocorrem a temperaturas inferiores à $\mathrm{T}_{\mathrm{g}}$, as quais são classificadas em ordem alfabética na seqüência em que ocorrem como $\beta, \gamma, \delta$, etc., a partir da $\mathrm{T}_{\mathrm{g}}$, que é classificada como transição $\alpha^{2}$. A técnica de DMA é especialmente útil na determinação dessas transições. As relaxações secundárias envolvem movimentos moleculares mais localizados, isto porque, abaixo da $\mathrm{T}_{\mathrm{g}}$ a mobilidade das cadeias é reduzida. Apesar desta limitação, estes movimentos moleculares podem ter um efeito significante sobre as propriedades macroscópicas de materiais poliméricos, que podem se manifestar como mudanças em propriedades mecânicas e físicas, tal como o módulo, a constante dielétrica e o coeficiente de expansão térmica. Os possíveis movimentos moleculares que ocorrem abaixo da $\mathrm{T}_{\mathrm{g}}$ foram classificados, em 1977, por Heijboer ${ }^{14}$ e são esquematizados na Figura 3. As relaxações do tipo "A", envolvem pequenos segmentos da cadeia principal, ocorrendo através de movimentos rotacionais em torno das ligações. Este tipo de movimento tem sido atribuído à relaxação $\beta$ no poli(cloreto de vinila) (PVC), à relaxação $\gamma$ no policarbonato $(\mathrm{PC})$, e às relaxações secundárias em polissulfonas e poliésteres . As relaxações do tipo "B" estão relacionadas aos grupos laterais que executam movimentos rotacionais em torno da ligação que os unem à cadeia principal. Ao realizar este movimento, o grupo move-se de um mínimo de energia potencial para outro, sem necessariamente realizar uma rotação completa, necessitando ainda de uma contribuição do movimento cooperativo da cadeia principal. Este tipo de movimento molecular está presente nas cadeias dos poli(metacrilatos de n-alquila). O movimento interno localizado de uma das unidades que faz parte de um grupo lateral da cadeia principal corresponde às relaxações do tipo " $C$ ". Novamente, os poli(metacrilatos de alquila) são um bom exemplo deste tipo de movimento molecular, sendo neste caso denominadas de relaxações $\gamma^{9}$.

Talvez um dos mais importantes efeitos destas relaxações secundárias nas propriedades macroscópicas seja a tenacidade ou a alta resistência ao impacto apresentada por alguns polímeros como, por exemplo, o policarbonato, cuja alta resistência ao impacto é usualmente associada à relaxação $\gamma$, que ocorre a baixas temperaturas. A relaxação principal ou transição vítrea do policarbonato (PC) ocorre em torno de $155^{\circ} \mathrm{C}$, a relaxação $\beta$ é observada em $80{ }^{\circ} \mathrm{C}$, e uma proeminente relaxação $\gamma$ ocorre a $-97{ }^{\circ} \mathrm{C}$. A relaxação $\beta$ no $\mathrm{PC}$ depende fortemente da história térmica do material, e é reduzida por tratamento térmico do material. Esta relaxação é atribuída à tensão causada pela orientação interna dos grupos laterais ou por defeitos que ocorrem durante o empacotamento no estado vítreo. A relaxação $\gamma$ é a que confere um comportamento mais interessante ao PC. Após várias tentativas para a atribuição desta transição aos movimentos internos das cadeias e grupos laterais ${ }^{15-19}$, Jones ${ }^{20}$ propôs um modelo molecular que combina movimentos intramoleculares com acoplamento intermolecular, como esquematizado na Figura $6^{20}$.
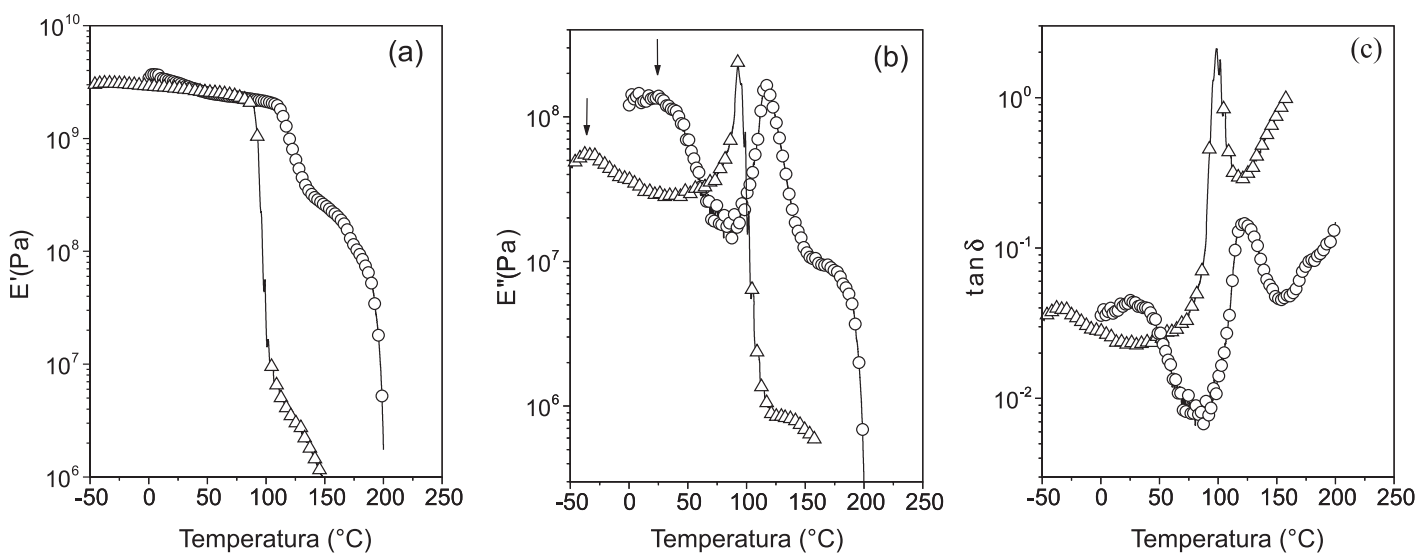

Figura 5. Curvas de DMA para o PET amorfo $(\Delta)$ e semicristalinos $(\bigcirc)$ : a) módulo de armazenamento (E') vs. temperatura, b) módulo de perda (E”) vs. temperatura e c) tan $\delta$ vs. temperatura 


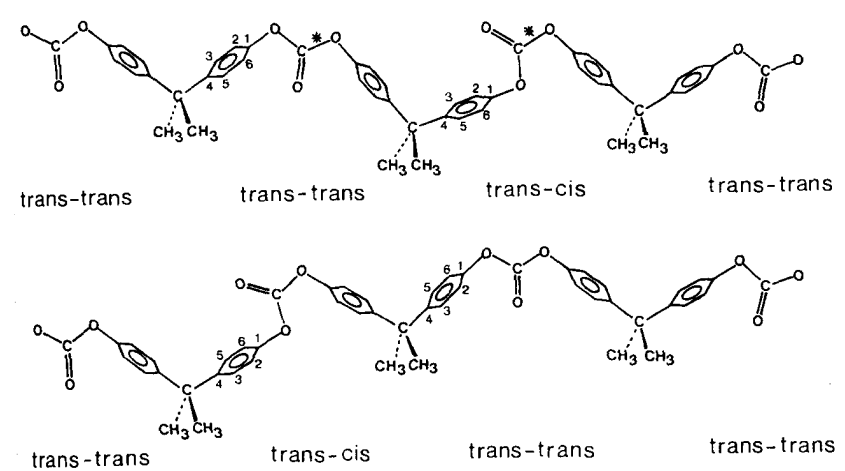

Figura 6. Policarbonato bisfenol A: a ligação C-O marcada com um asterisco indica o ponto de rotação. $O$ anel fenila numerado sofre uma inversão quando a ligação $\mathrm{C}$-O realiza o movimento de rotação. Reproduzida da ref. 20, com permissão da ACS

A cadeia de policarbonato sofre mudanças conformacionais envolvendo rotações de ligações de dois grupos carbonatos vizinhos, que mudam da conformação trans-cis para uma trans-trans. Este movimento requer a rotação em torno da ligação $\mathrm{C}-\mathrm{O}$ marcada com asterisco, com uma inversão do grupo fenila em torno do eixo $\mathrm{C}_{1} \mathrm{C}_{4}$, resultando em uma flutuação de volume livre como consequiência da translação do grupo bisfenol A. Estes processos de relaxação se difundem ao longo da cadeia com sucessiva mudança conformacional constituindo um mecanismo de rápida dissipação de tensão e, portanto, rápida absorção de energia durante o impacto. Este modelo é consistente com os requisitos geométricos obtidos por ressonância magnética nuclear (RMN) e com as curvas de módulo de perda obtidas através de ensaios dinâmico-dielétrico e dinâmico-mecânico9 .

As relaxações secundárias são importantes também em polímeros metacrílicos. Em estudos do comportamento dinâmico-mecânico desses polímeros, Hoff et al. ${ }^{21}$ identificaram regiões de relaxação $\beta$ e $\gamma$, além da relaxação principal $\alpha$. Estes autores notaram que a relaxação $\beta$ poderia ser atribuída ao rearranjo rotacional dos grupos laterais $\mathrm{COO}$ em torno da cadeia principal, e que a magnitude desta relaxação diminuía, chegando mesmo a desaparecer com o aumento do grupo lateral na série metila, etila, n-propila e n-butila. Portanto, a relaxação $\beta$ mostra-se dependente do comprimento do segmento lateral ${ }^{21}$. O fato desta relaxação desaparecer em metacrilatos contendo grupos laterais nalquila com alto número de carbonos é atribuído à sobreposição desta relaxação e da relaxação $\alpha$ (transição vítrea). A intensidade da relaxação principal $\alpha$ diminui com o aumento do comprimento do grupo lateral, devido ao maior volume livre associado a este e também ao impedimento estérico causado pelas cadeias vizinhas9. Embora seja amplamente aceito que a relaxação $\beta$ em polímeros metacrílicos esteja associada à rotação intramolecular do grupo COOR, onde $\mathrm{R}$ depende da série homóloga em particular, também há evidências que esta relaxação esteja associada a interações intermoleculare ${ }^{22-24}$.

A relaxação $\gamma$ em metacrilatos e clorometacrilatos de n-propila, n-butila e n-alquilas contendo um número maior de carbonos foi identificado por Hoff et al. ${ }^{21}$ a cerca de $-150{ }^{\circ} \mathrm{C}$ a $1 \mathrm{~Hz}$. Dentre os polímeros destas séries homólogas aqueles contendo grupos laterais mais curtos não apresentaram relaxações na região $\gamma^{9}$. Este comportamento foi explicado considerando-se que a relaxação nesta região é devida à flexibilidade de grupos alquila mais longos, como foi observado na série homóloga ${ }^{9}$. Em metacrilatos cíclicos pode-se ainda observar a relaxação $\delta^{9}$.

As relaxações secundárias podem ser afetadas pela cristalinidade. Nas curvas E" x T e tan $\delta$ x T para o PET amorfo e semicristalino, Figuras $5 \mathrm{~b}$ e $5 \mathrm{c}$, é possível ver-se o deslocamento da relaxação secundária em torno de $-30{ }^{\circ} \mathrm{C}$ (PET amorfo) para aproximadamente
$+30{ }^{\circ} \mathrm{C}$ (PET semicristalino). A relaxação secundária do PET é atribuída à presença da fase cristalina e inibe a relaxação secundária da fase amorfa do PET, deslocando-a em $60{ }^{\circ} \mathrm{C}$ para temperaturas mais altas. Também é observado o alargamento da relaxação secundária para o PET semicristalino.

\section{Avaliação da miscibilidade em blendas poliméricas}

A miscibilidade ou a extensão da segregação de fases em blendas pode ser avaliada por DMA. Classicamente, blendas são definidas como uma mistura física entre dois ou mais homopolímeros ou copolímeros $^{13}$. Outras definições podem ser encontradas na literatu$\mathrm{ra}^{25,26}$. Um dos critérios para a avaliação da miscibilidade em blendas é a análise da transição vítrea. Sob este critério, uma blenda é considerada miscível quando apresenta uma única transição vítrea situada em uma faixa de temperatura intermediária às transições dos componentes puros. A condição para a aplicação deste critério é que haja uma diferença de pelo menos $20^{\circ} \mathrm{C}$ entre as temperaturas de transição vítrea dos componentes da blenda ${ }^{13}$. Uma blenda parcialmente miscível apresenta duas ou mais transições vítreas, correspondentes às diferentes fases, deslocadas em relação à $\mathrm{T}_{\mathrm{g}}$ dos componentes puros. Neste caso, cada fase é constituída de uma mistura miscível contendo diferentes composições. Por outro lado, blendas imiscíveis apresentam transições vítreas características aos componentes pu$\operatorname{ros}^{13,25,26}$.

A transição vítrea é acompanhada por uma variação de capacidade calorífica de poucos décimos de $\mathrm{J} / \mathrm{g}{ }^{\circ} \mathrm{C}$ e por uma variação acentuada do módulo de armazenamento, da ordem de $10^{3} \mathrm{~Pa}$ no caso de polímeros amorfos. Estas características da transição vítrea tornam a técnica de DMA especialmente sensível para a sua detecção em comparação a técnicas calorimétricas, por exemplo. Em alguns casos, como em blendas ou compósitos, nos quais um dos componentes está presente em baixa concentração, a sua transição vítrea muitas vezes só pode ser detectada por análise dinâmico-mecânica ${ }^{1}$.

Além da possibilidade de detecção de transições de componentes em baixas concentrações em blendas, a análise da largura das transições permite obter informações sobre a micro-heterogeneidade das fases. No caso de misturas, o termo micro-heterogeneidade refere-se também à existência de vizinhanças com composição diferenciada para os segmentos de uma mesma cadeia polimérica ${ }^{13}$.

Exemplos de DMA de blendas miscível e imiscível são mostrados na Figura 7. A blenda de poli(álcool vinílico) PVAL e poli(vinilpirrolidona) (PVP) é miscível e apresenta micro-heterogeneidade em algumas composições. Na Figura 7a são mostradas as curvas do módulo de perda em função da temperatura para essas blendas obtidas em diferentes composições ${ }^{27}$. Os máximos dos picos das curvas de log E" x T observados para os homopolímeros, PVAL e PVP, ocorrem a 75 e $184{ }^{\circ} \mathrm{C}$, respectivamente, e são atribuídos à transição vítrea desses polímeros. O PVAL é um polímero semicristalino, cuja fase cristalina funde em torno de $250^{\circ} \mathrm{C}$. As cadeias de PVAL na interface cristalina-amorfa apresentam mobilidade menor do que teriam se estivessem na fase amorfa. Portanto, relaxam a temperaturas superiores à $\mathrm{T}_{\mathrm{g}}$ do PVAL. No caso das blendas PVAL/ PVP a fase amorfa constituída da mistura miscível, apresenta uma única transição vítrea indicada pela seta preenchida, que ocorre entre as transições vítreas observadas para os homopolímeros, de acordo com a composição. As transições que ocorrem a temperaturas inferiores à $\mathrm{T}_{\mathrm{g}}$, nas composições entre 50 e $80 \%$ em massa de PVAL (setas vazias), são atribuídas à relaxação dos segmentos das cadeias do PVAL que se situam na interface, fase cristalina do PVAL e fase amorfa da mistura homogênea PVAL/PVP.

As blendas de PMMA e poli(butadieno-co-acrilonitrila) (NBR) são imiscíveis em toda a faixa de composição, como pode ser obser- 
(a)

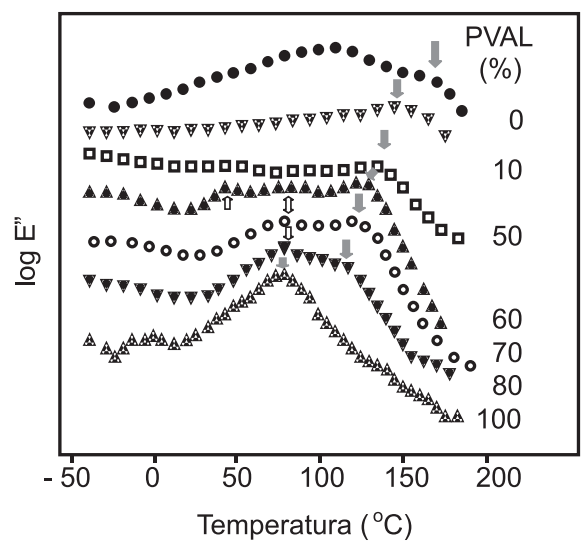

(b)

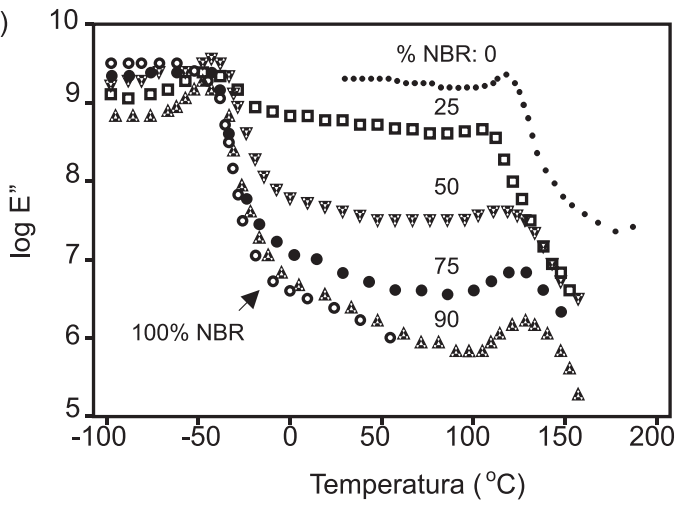

(c)

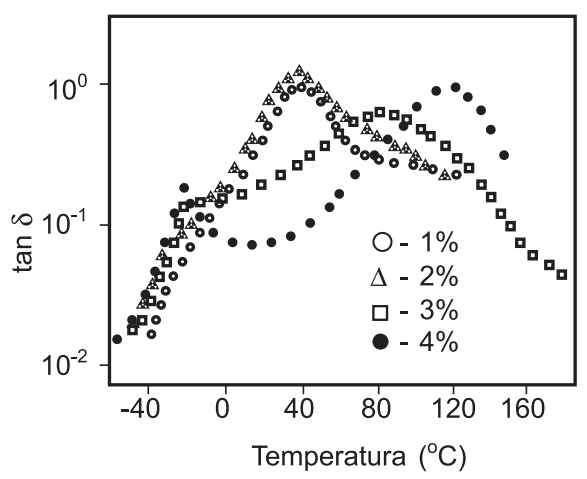

Figura 7. Curvas do módulo de perda, E" $x$ T para blendas: (a) miscíveis PVAL/PVP e (b) imiscíveis PMMA/NBR; (c) curvas de tan $\delta x$ T para semiIPNs parcialmente miscíveis de PVME e poli(estireno-co-anidrido maleico)

vado nas curvas de $\log$ E” x T, Figura 7b, na qual a temperatura de transição vítrea das misturas é coincidente com a $\mathrm{T}_{\mathrm{g}}$ dos homopolímeros $^{28}$.

Exemplos de misturas parcialmente miscíveis são as semi-IPNs ${ }^{29}$. Em semi-IPNs de poli(estireno-co-anidrido maleico), contendo 4,8\% em mol de anidrido, e poli(vinil metil éter) (PVME), a imiscibilidade foi induzida através da reticulação do poli(estireno-co-anidrido maleico) e a sua extensão foi avaliada por análise dinâmico-mecâni$\mathrm{ca}^{30}$. As curvas de $\tan \delta$ em função da temperatura obtidas para as semi-IPNs (50/50), contendo diferentes teores de reticulante são apresentadas na Figura 7c. A baixos teores de reticulante (1 e 2\%) um único pico com máximo em $35^{\circ} \mathrm{C}$ é observado, o qual é atribuído à transição vítrea do material, indicando miscibilidade deste sistema. Quando 3\% do reticulante é adicionado à semi-IPN, observa-se um alargamento da relaxação principal, e a $4 \%$ de reticulante, pode ser vista uma completa separação de fases, observando-se duas relaxações principais ocorrendo nas mesmas regiões de transição vítrea dos componentes puros PVME e poli(estireno-co-anidrido maleico), a -30 e $120{ }^{\circ} \mathrm{C}$, respectivamente ${ }^{30-31}$.

A miscibilidade parcial de blendas de poli(metacrilato de metila) e poli(epicloridrina-co-óxido de etileno) foi observada através de análise dinâmico-mecânica, enquanto técnicas calorimétricas indicavam a existência de apenas uma transição vítrea larga ${ }^{32}$.

Estudos dinâmico-mecânicos de resinas de poliéster insaturado, utilizando como agente de cura o monômero estireno, mostraram que a teores de estireno superiores a $18 \%$ ocorre segregação de fases, identificando-se duas transições ocorrendo em torno de 50 e $66{ }^{\circ} \mathrm{C}$, que sugerem que a resina é constituída por pelo menos duas fases, uma contendo o poliéster insaturado reticulado e uma rica em poliestireno $^{33}$.

O comportamento dinâmico-mecânico permite ainda a avaliação de mudanças morfológicas e estruturais causadas pelo processamento e pela adição de aditivos, desde que se conheçam os componentes desses sistemas ${ }^{5,10}$. Blendas imiscíveis podem não apresentar boas propriedades mecânicas, devido à má dispersão e pobre adesão das fases. Tecnologicamente, o caminho para resolver estas questões é a compatibilização $0^{26,34,35}$. Há diferentes formas de se promover a compatibilização, sendo uma das mais usadas a compatibilização in situ, na qual ocorre a formação de um copolímero de enxertia constituído pelos diferentes segmentos dos componentes da blenda, durante a preparação desta por mistura mecânica no estado fundi$\mathrm{do}^{34,35}$. A técnica de DMA tem sido utilizada com sucesso para verificar a formação do copolímero compatibilizante. Por exemplo, em blendas de poliamida 6 (PA6) e poli(epicloridrina-co-óxido de etileno) obtidas no estado fundido, ocorre a formação de um copolímero de enxertia, constatado através de ensaios de solubilidade seletiva e de análise dinâmico-mecânica ${ }^{36}$. A curva do módulo de perda em função da temperatura obtida para as blendas contendo $20 \%$ de elastômero é mostradas na Figura 8. A temperatura de transição vítrea da PA6, que ocorre em cerca de $57^{\circ} \mathrm{C}$, é deslocada na blenda para cerca de $50{ }^{\circ} \mathrm{C}$. Observa-se um ombro em torno de $25{ }^{\circ} \mathrm{C}$ na blenda, que não ocorre nos polímeros puros, o qual foi atribuído à presença do copolímero de enxertia formado durante a obtenção do material.

Blendas de PMMA e poliamida 6, 9/9 e 12 foram avaliadas por meio de microscopia eletrônica de transmissão e análise dinâmicomecânica $^{37}$. As blendas são imiscíveis, apresentando duas transições vítreas. Para a estimativa da extensão de segregação de fases $(\alpha)$ foi utilizada a equação empírica proposta por Lipatov ${ }^{38}$. Os resultados de DMA indicaram que as blendas PMMA/PA-6, mesmo sendo imiscíveis, apresentam um certo grau de interpenetração de fases.

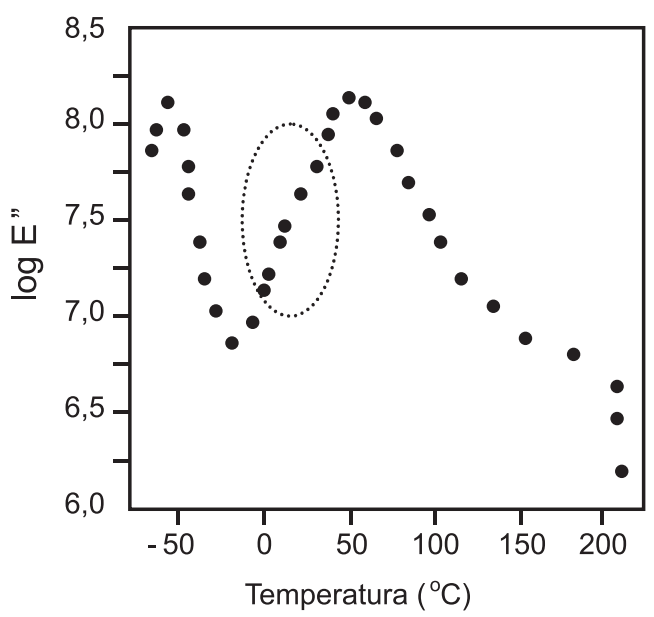

Figura 8. Módulo de perda, E” x T para blenda de poliamida-6 e poli(epicloridrina-co-óxido de etileno) (80/20) 


\section{Módulo de perda (E”) vs. fator de perda $(\tan \delta)$}

Sperling et al. ${ }^{39}$ usando a teoria de contribuição de grupos propuseram que a energia mecânica dissipada por um determinado polímero durante um ciclo em um experimento dinâmico-mecânico é dependente dos grupos presentes na cadeia polimérica, e que estes grupos absorvem determinadas quantidades de energia. Segundo essa teoria, a área sob a curva E" x T na região de transição vítrea (LA), permite uma análise quantitativa da contribuição dos diferentes grupos constituintes da cadeia polimérica na dissipação de energia mecânica.

A área LA na região de transição vítrea pode, então, ser descrita como o somatório das contribuições dos $i$ grupos químicos:

$L A=\sum_{i=1}^{n} \frac{(L A)_{i} M_{i}}{M}=\sum_{i=1}^{n} \frac{C_{i}}{M}$

onde $\mathrm{M}_{\mathrm{i}}$ é a massa molar do iésimo grupo na unidade repetitiva, $\mathrm{M}$ a massa molar do polímero, $\mathrm{C}_{\mathrm{i}}$ - definida como constante de perda molar para o iésimo grupo, (LA) - a contribuição em área do iésimo grupo sob a curva do módulo de perda em função da temperatura e $n$ representa o número de grupos capazes de absorver energia no polímero. Entretanto, a Equação (19) considera apenas a composição, não importando o comportamento de fases do sistema, as possíveis interações entre os diferentes grupos químicos e as diferenças morfológicas. De acordo com esta teoria, enquanto a curva do módulo de perda em função da temperatura, similarmente à espectroscopia na região de infravermelho, é uma função da estrutura molecular de um polímero e a área sob esta curva está relacionada à concentração das espécies envolvidas, as propriedades de "damping" de um sistema polimérico são relacionadas às suas estruturas morfológica e química, e podem ser expressas pela área sob a curva de $\tan \delta$ em função da temperatura ${ }^{40}$.

Esta teoria se aplica na descrição de materiais poliméricos utilizados em isolamento acústico, abrangendo desde isolantes acústicos para aviões até sua utilização na construção civil. Uma forma de avaliar sua eficiência é através da análise da área e da largura dos picos das curvas de $\tan \delta \mathrm{x} \mathrm{T}$, associados normalmente à transição vítrea. Quanto mais largo o pico tan $\delta$ x T para uma determinada relaxação, mais largo será o espectro de relaxação do material, sendo que uma faixa mais ampla de frequiência poderá ser absorvida por este material. $\mathrm{O}$ trabalho de Hourston et al. $^{40}$ exemplifica bem as informações que as áreas sob as curvas de módulo de perda e tan $\delta$ fornecem sobre um dado sistema. Os autores determinaram as áreas sob as curvas de E" e tan $\delta$ em função da temperatura para redes interpenetrantes poliméricas compostas por poliuretanas termoplásticas e poli(metacrilato de etila) (PEMA). A área sob a curva de E" aumentou gradualmente com o aumento do teor de PEMA, refletindo o aumento da concentração de grupos com maior capacidade de converter energia mecânica em térmica, enquanto que, a área sob a curva de $\tan \delta$ atingiu valor máximo a $30 \%$ em massa de PEMA, composição esta que apresentou a melhor relação entre a energia dissipada e a energia armazenada, ou seja, o máximo "damping", correspondendo à composição da IPN que apresentou o melhor desempenho como isolante acústico.

\section{Correlação entre a curva de “damping” e as propriedades mecânicas}

Os estudos que buscam correlacionar o comportamento dinâmico-mecânico e a resistência ao impacto tiveram início por volta de $1950^{41}$. A questão a ser considerada era se havia correlação entre as propriedades mecânicas de polímeros e o comportamento dinâmico- mecânico na região de temperatura de solicitação do material que, em geral, para polímeros vítreos está abaixo da temperatura de transição vítrea.

Alguns autores como, por exemplo, Bohn, Illers, Kilian e Kosfeld (apud ref. 41) acreditavam que a existência de uma relaxação secundária abaixo da temperatura de transição vítrea poderia ser a responsável pela tenacidade encontrada em alguns polímeros, tomando como principais exemplos para isto o policarbonato (PC) e o poliestireno de alto impacto (HIPS). Nestes dois materiais há a ocorrência de uma relaxação à baixa temperatura, sendo o caso do PC já citado, enquanto que no HIPS essa relaxação é relacionada à transição vítrea da fase borracha e sua temperatura pode variar de $-100 \mathrm{a}-20^{\circ} \mathrm{C}$, dependendo principalmente do tipo da borracha utilizada. Entretanto, esta hipótese falha para polímeros acrílicos, os quais apesar de apresentarem em geral mais de uma relaxação secundária a temperaturas inferiores à de transição vítrea, não apresentam altos valores de resistência ao impacto quando comparados ao PC e ao HIPS. Vincent (apud ref. 41) acreditava não haver uma correlação entre a transição frágil-dúctil e as relaxações moleculares obtidas por ensaios dinâmico-mecânicos.

Ainda em 1968, em um estudo envolvendo uma série de polímeros acrílicos, poli(óxido de fenileno) (PPO) e PC entre outros, Heijboer ${ }^{42}$ concluiu que quando as relaxações secundárias estão associadas a movimentos isolados de grupos laterais da cadeia principal, como ocorre em polímeros acrílicos, estas não contribuem para a tenacidade do material, observando-se o típico comportamento de material rígido e quebradiço. Porém, se as relaxações envolvem não apenas os movimentos de grupos laterais, mas também um pequeno movimento da cadeia principal, a probabilidade de um aumento de resistência ao impacto é alta, que é justamente o que ocorre nos casos do PC e do PPO.

Em 1971, Keskkula et al. ${ }^{43}$ estudaram o comportamento dinâmico-mecânico e de resistência ao impacto de HIPS e ABS, obtidos de diferentes fornecedores. Foram determinadas as áreas sob a curva de $\tan \delta$ em função da temperatura, bem como da altura do pico de tan $\delta$, na região de relaxação da fase borracha. Os autores relacionaram estes resultados à resistência ao impacto destes materiais, verificando não haver correlação entre as propriedades de "damping" e as propriedades de resistência ao impacto. Porém, neste mesmo trabalho, blendas de PS e poli(estireno-co-butadieno) contendo 5, 10 e $20 \%$ em massa do elastômero obtidas por solução, foram comparadas às respectivas blendas obtidas por mistura mecânica. Nestas séries constatou-se que havia um aumento tanto na área como na altura dos picos de tan $\delta$ com o aumento do teor da fase elastomérica. Também foi possível se estabelecer uma correlação entre as propriedades de "damping" e as de resistência ao impacto, observando-se um aumento destas com o aumento no teor de elastômero.

Karger-Kocsis e Kuleznev ${ }^{44}$, em 1982, encontraram uma relação linear entre o "damping" e a resistência ao impacto em blendas de polipropileno e EPDM.

Um importante ponto a ser lembrado é que a correlação entre as transições secundárias observadas por meio da técnica dinâmicomecânica e a resistência ao impacto, geralmente, não consideram que as transições secundárias são medidas em condições de baixa deformação, região de viscoelasticidade linear, enquanto que a resistência ao impacto é medida na região de viscoelasticidade nãolinear.

Estes exemplos mostram como nem sempre é possível se fazer uma associação direta entre os resultados obtidos por ensaios mecânicos e aqueles obtidos por experimentos dinâmico-mecânicos. A relação entre essas propriedades é principalmente dependente da natureza do material em questão, não sendo possível se estabelecer uma regra simples para fixar esta correlação. 


\section{CONCLUSÃO}

A utilização da técnica dinâmico-mecânica em sistemas poliméricos traz importantes contribuições a respeito das estruturas química e morfológica desses sistemas, sendo possível se determinar além das principais relaxações, como a transição vítrea, fusão e cristalização, relaxações de menor magnitude, como é o caso das relaxações secundárias.

É possível inferir sobre as mudanças no ambiente químico de um determinado grupo quando há, por exemplo, variação de composição em blendas, adição de diferentes teores de aditivos ou cargas, através da análise do perfil ou da área sob a curva de E" x T.

Informações sobre as propriedades acústicas podem ser obtidas através da análise da área sob a curva do $\tan \delta$ versus temperatura, no caso de materiais utilizados como isolantes acústicos.

A associação do comportamento dinâmico-mecânico com o comportamento mecânico de um material segue uma regra complexa, não podendo ser generalizado a partir do estudo de alguns sistemas específicos.

\section{REFERÊNCIAS}

1. Wunderlich, B. Em Thermal Characterization of Polymer Materials; Turi, E. A., ed.; $2^{\text {nd }}$.ed., Academic Press Inc.: New York, 1997, vol. 1, p. 305.

2. Wetton, R. E. Em Developments in Polymer Characterization; Dawkins, J. V., ed.; Elsevier Applied Sci. Publishers: London, 1986, p. 179

3. McCrum, N. G.; Buckley, C. P.; Bucknall, C. B.; Principles of Polymer Engineering, $2^{\text {nd }}$ ed., Oxford University Press Inc.: New York, 1997, cap. 4.

4. Brown, M. E.; Introduction to Thermal Analysis, $1^{\text {st }}$ ed.; Chapman and Hall Ltd.: New York, 1988, cap.8.

5. Nielsen, L. W.; Mechanical Properties of Polymers and Composites; Marcel Dekker, INC.: New York, 1974, vol. 1 e 2.

6. Murayama, T. Em Encyclopedia of Polymer Science and Engineering; Mark, H. F.; Bikales, N. M.; Overberger, C. G.; Menges, G.; Kroschwits, J. I., eds.; $2^{\text {nd }}$ ed., John Wiley \& Sons: New York, 1988, vol. 5, p. 299.

7. Menard, P. K.; Dynamic Mechanical Analysis: A Practical Introduction, CRC Press LLC: New York, 1999.

8. Ferry, J. D.; Viscoelastic Properties of Polymers, John Wiley \& Sons: New York, 1980 .

9. Ward, I. M.; Mechanical Properties of Solid Polymers, $2^{\text {nd }}$ ed., John Wiley \& Sons: New York, 1983

10. Hutchinson, J. M. Em The Physics of Glassy Polymers; Haward, R. N.; Young, R. J., eds.; $2^{\text {nd }}$ ed.; Chapman and Hall: London, 1997, p. 85.

11. Gradin, P.; Howgate, P. G.; Seldén, R.; Brown, R. Em Comprehensive Polymer Science; Allen, G.; Bevington, J. C.; Booth, C.; Price, C., eds.; $1^{\text {st }}$ ed.; Pergamon Press: New York, 1989, vol. 2, p. 533
12. Mark, J. E.; Eisenberg, A.; Graessley, W. W.; Mandelkern, L.; Samulski, E. T.; Koenig, J. L.; Wignall, G. D., eds.; Physical Properties of Polymers, $2^{\text {nd }}$ ed., American Chemical Society: Washington, 1993.

13. Olabisi, O.; Robeson, L. M.; Shaw, M. T., eds.; Polymer-Polymer Miscibility, Academic Press: New York; 1979.

14. Heijboer, J.; Int. J. Polym. Mater. 1977, 6, 11.

15. Jho, J. Y.; Yee, A. F.; Macromolecules 1991, 24, 1905

16. Yee, A. F.; Smith, S. A.; Macromolecules 1981, 14, 54.

17. Schaefer, J.; Steijskal, E. O.; Perchak, D.; Skolnick, J.;Yaris, R.; Macromolecules 1985, 18, 368.

18. Yee, A. F.; Polym. Eng. Sci. 1977, 17, 213.

19. Floudas, G.; Higgins, J. S.; Meier, G.; Kremer, F.; Fischer, E. M.; Macromolecules 1993, 26, 1676.

20. Jones, A. A.; Macromolecules 1985, 18, 902

21. Hoff, E. A. W.; Robinson, D. W.; Willbourn, A. H.; J. Polym. Sci. 1955, 18,161 .

22. Williams, G.; Trans-Faraday Soc. 1966, 62, 2091.

23. Dionisio, M. S.; Moura-Ramos, J. J.; Williams, G.; Polymer 1994, 35, 1705.

24. Diaz-Calleja, R.; Devine, I.; Gargallo, L.; Radic, D.; Polymer 1994, 35, 151.

25. Utracki, L. A.; Polymer Alloys and Blends: Thermodynamics and Rheology, Hanser Publishers: New York, 1990, p. 1.

26. Paul, D. R.; Bucknall, C. B.; Polymer Blends, John Wiley \& Sons: New York, 1999.

27. Cassu, S. N.; Felisberti, M. I.; Polymer 1999, 40, 4845

28. Santos, L. E. P.; Dissertação de Mestrado, Universidade Estadual de Campinas, Brasil, 1995.

29. Kempler, D.; Sperling, L. H.; Utracki, L. A.; Interpenetrating Polymer Networks, Advances in Chemistry Series: Washington, DC, 1994, p. 234.

30. Felisberti, M. I.; Ph. Thesis, Albert-Ludwig-Universität, Alemanha, 1990.

31. Felisberti, M. I.; Müller, G.; Stadler, R.; Polym. Mater Sci. Eng., Proc. of ACS Div. PMSE, 1990, vol. 62, p. 659

32. Rocha, S. M.; Tese de Doutorado, Universidade Estadual de Campinas, Brasil, 1998.

33. Sanchez, E. M. S.; Zavaglia, C. A. C.; Felisberti, M. I.; Polymer 2000, 41, 765.

34. Di Lorenzo, M. L.; Frigione, M.; J. Polym. Eng. 1997, 17, 429.

35. Koninig, C.; Van Duin, M.; Pagnoulle, C.; Jerome, R.; Prog. Polym. Sci. 1998, 23, 707.

36. Costa, S. C. G.; Felisberti, M. I.; J. Appl. Polym. Sci. 1999, 72, 1835

37. Carone, E. Jr.; Felisberti, M. I.; Nunes, S. P.; J. Mater. Sci. 1998, 33, 3729.

38. Lipatov, Y. S. Em Interpenetrating Polymer Network; Klempson, D.; Sperling, L. H.; Utracki, L. A., eds.; ACS: Washington, DC, 1994.

39. Chang, M. C. O.; Thomas, D. A.; Sperling, L. H.; J. Polym. Sci., Part B: Polym. Phys. 1988, 26, 1627.

40. Hourston, D. J.; Schäfer, F-U.; High Perform. Polym. 1996, 8, 19.

41. Boyer, R. F.; Polym. Eng. Sci. 1968, 8, 161

42. Heijboer, J.; J. Polym. Sci., Part C: Polym. Sym. 1968, 16, 3755.

43. Keskkula, H.; Turley, S. G.; Boyer, R. F.; J. Appl. Polym. Sci. 1971, 15, 351 .

44. Karger-Kocsis, J.; Kuleznev, V. N.; Polymer 1982, 23, 699. 